\title{
La halitosis: revisión de la literatura. Segunda parte
}

\author{
Foglio Bonda PL, Rocchetti V, Migliario M, Giannoni M*
}

\section{RESUMEN}

La halitosis es un trastorno capaz de condicionar la calidad de vida, particularmente en lo que atañe a las relaciones sociales. Mediante una atenta revisión de la literatura se han puesto de relieve las hipótesis etiológicas, tanto orales como extraorales, de la halitosis y el correspondiente enfoque diagnóstico-terapéutico por parte del odontólogo y del higienista dental.

Palabras clave: Halitosis, higiene oral, diagnóstico y tratamiento.

\section{SUMMARY}

Halitosis is a problem that may affect the quality of life, social relations in particular. Through a careful literature review, aetiological hypotheses, both oral and extraoral, on halitosis are highlighted as well as the dianostic and therapeutic approach that should be adopted by the dentist and the dental hygienist.

Key words: Halitosis, oral-hygiene, diagnosis and treatmet.

Fecha de recepción: Junio 2007.

Aceptado para publicación: Julio 2007.

Universidad del Piamonte Oriental, Licenciatura en Higiene Dental, Presidente: Prof. P. L. Foglio Bonda.

* $\quad$ Universidad de L'Aquila, Licenciatura en Higiene Dental, Presidente: Prof. M. Giannoni.

Publicado en DOCTOR OS 2007, núm. 5; Traducido por Pablo Herrero Hernández.

Foglio Bonda PL, Rocchetti V, Migliario M, Giannoni M. La halitosis: revisión de la literatura. segunda parte. Av.

Odontoestomatol 2008; 24 (2): 167-175.

\section{HALITOSIS DE ORIGEN EXTRAORAL Y SISTÉMICO}

\section{Enfermedades sistémicas}

Entre las causas extraorales de halitosis deben incluirse algunas patologías sistémicas graves que en fase de descompensación se asocian a trastornos metabólicos que pueden proporcionar olores característicos al aliento de los pacientes.

Entre estas patologías destacan, por su frecuencia, la diabetes mellitus, la insuficiencia renal crónica y las hepatopatías graves $(1,14,44,45)$ (tabla 5).
La diabetes mellitus puede caracterizarse por el olor típico del aire espirado (aliento cetónico) $(1,20)$, similar al de las manzanas podridas (46), debido al paso por la zona alveolar pulmonar de acetona, acetoacetato y $\beta$-hidroxibutirato, producidos en exceso como consecuencia de un metabolismo glucídico anómalo $(1,2,13)$. La emisión por la cavidad oral de aliento cetónico se considera un síntoma típico de diabetes descompensada, si bien sólo pocos casos se diagnostican de esta manera (8).

En la insuficiencia renal crónica que cursa con hiperuricemia, el aliento del paciente puede presentar el olor característico a pescado podrido (aliento urémi- 


\section{TABLA 5.- PRINCIPALES PATOLOGÍAS SISTÉMICAS RESPONSABLES DE LA PRODUCCIÓN DE MAL OLOR}

\begin{tabular}{|l|l|}
\hline Patología & $\begin{array}{l}\text { Compuestos volátiles } \\
\text { identificados }\end{array}$ \\
\hline $\begin{array}{l}\text { Diabetes mellitus } \\
\text { (aliento cetónico) }\end{array}$ & $\begin{array}{l}\text { Acetona, acetoacetato, } \\
\beta \text {-hidroxibutirato }\end{array}$ \\
\hline $\begin{array}{l}\text { Insuficiencia renal } \\
\text { (aliento urémico) }\end{array}$ & $\begin{array}{l}\text { Dimetilamina }\left(\mathrm{CH}_{3}\right)_{2} \mathrm{NH}, \\
\text { trimetilamina }\left(\mathrm{CH}_{3}\right)_{3} \mathrm{~N}\end{array}$ \\
\hline $\begin{array}{l}\text { Cirrosis hepática } \\
\text { (foetor hepaticus) }\end{array}$ & $\begin{array}{l}\mathrm{C}_{2}-\mathrm{C}_{3} \text { ácidos alifáticos, } \\
\text { metilmercaptano }\left(\mathrm{CH}_{3} \mathrm{SH}\right), \\
\text { sulfuro de dimetilo }\left(\mathrm{CH}_{3} \mathrm{SCH}\right)\end{array}$ \\
\hline
\end{tabular}

co) causado por la exhalación pulmonar de compuestos químicos como la dimetilamina y la trimetilamina (45).

El paciente aquejado de cirrosis hepática o de otras formas de insuficiencia hepática grave puede presentar lo que se denomina foetor hepaticus, resultado de la eliminación corporal y respiratoria de ácidos alifáticos de cadena corta y de compuestos de sulfuro como el metilmercaptano, el etanol y el sulfuro de dimetilo $(1,2,46)$. El olor es del tipo fecaloide, y en los casos de insuficiencia hepática terminal se percibe en toda la habitación en la que se encuentra el paciente (46).

Hay también enfermedades congénitas del metabolismo, como la trimetilaminuria o síndrome del olor a pescado, que pueden producir olores desagradables del aire espirado (2). En la sangre, en la orina y en el aire espirado de pacientes aquejados de dicha patología se detectan, en efecto, cantidades anómalas de trimetilamina, un compuesto volátil que aporta a las secreciones corporales y al aire espirado un olor a pescado podrido similar al de los pacientes con insuficiencia renal crónica (1).

\section{Enfermedades otorrinolaringológicas, broncopulmonares y gastrointestinales}

La halitosis puede formar parte del cortejo sintomatológico de enfermedades que afectan a órganos y aparatos que comunican con el exterior mediante la cavidad oral (46).

En efecto, los fenómenos inflamatorios, infectivos o neoplásicos de los aparatos respiratorio y gastrointestinal que cursan con presencia de sustratos proteicos como exudados, secreciones patológicas, pus o tejidos necróticos, favorecen el desarrollo de microorganismos putrefactivos, con el consiguiente foetor ex ore. Por este motivo se incluyen entre las causas extraorales de halitosis las afecciones infectivo-inflamatorias otorrinolaringológicas y, entre éstas, además de las sinusitis agudas y crónicas, las amigdalitis agudas y crónicas con exudado purulento, la presencia de criptas tonsilares de profundidad anormal $(1,20)$ y también las flogosis a nivel de las vegetaciones adenoideas, a menudo responsables de exudados purulentos continuados en la faringe, con la consiguiente contaminación del dorso lingual. En algunos individuos se forman, además, en las criptas tonsilares, unas concreciones denominadas tonsilolitos que pueden migrar a la superficie lingual. Estas concreciones, similares a piedrecitas, suelen alcanzar unos cuantos milímetros de longitud; son porosas, de color blanco o amarillo y malolientes, especialmente al aplastarse (8). No obstante, la función de las amígdalas en la génesis de la halitosis no resulta del todo clara, aun cuando, en los niños, resulte común un mal olor transitorio asociado a infecciones tonsilares (6).

Una posible causa de halitosis de origen nasal es la presencia de pólipos o de cuerpos extraños en la nariz (2). Rosenberg (8) ha puesto de relieve que una de las aportaciones principales a la posibilidad de generar halitosis por parte de las biopelículas del cubrimiento lingual está a cargo de lo que se denomina "goteo postnasal", es decir el goteo desde las coanas de secreciones mucosas ricas en proteínas que se depositan en el tercio posterior de la lengua para ser sucesivamente metabolizadas por los microorganismos presentes en el dorso lingual $(1,8)$. Entre las enfermedades broncopulmonares, las bronquiectasias, los abscesos pulmonares, las neoplasias pulmonares y, con menor frecuencia, las bronquitis, las pulmonías y la tuberculosis contribuyen al desarrollo de la halitosis $(1,13)$.

La búsqueda de partículas volátiles presentes en el aire espirado también puede desempeñar una fun- 
ción diagnóstica. En efecto, en pacientes aquejados de carcinoma broncógeno se han llevado a cabo estudios pormenorizados, basados en la utilización de técnicas de determinación gascromatográfica para la detección de compuestos volátiles. En dichos pacientes se han encontrado compuestos como acetona, metiletilcetona, n-propanol y concentraciones elevadas y características de o-toluidina no detectables en los grupos de control (1, 2, 20, 45).

Contrariamente a lo que se suele pensar, la halitosis de origen gastrointestinal es extremadamente infrecuente, ya que en los sujetos sanos el esófago está normalmente colapsado y el esfínter cardial cerrado; por consiguiente, si bien es verdad que episodios de eructación pueden provocar la presencia ocasional de mal olor en la cavidad oral, la posibilidad de un paso continuo de aire procedente del aparato gastrointestinal no es realista $(1,8)$.

Entre las patologías gastrointestinales que generan foetor ex ore se señalan las enfermedades esofágicas con incontinencia esfinteriana gastroesofágica y las enfermedades neoplásicas esofágicas y gástricas $(1,20)$.

Durante los últimos años se han llevado a cabo algunos estudios con el fin de correlacionar la infección gástrica por Helicobacter pylori (HP) y la halitosis $(1,2)$. Norfleet ha confirmado dicha asociación demostrando su desaparición después de tratamiento médico de la infección por HP (48).

\section{Hábitos alimentarios}

Es universalmente sabido que el consumo de alimentos como el ajo, la cebolla, el puerro y algunas especias puede causar la presencia de olores desagradables del aliento durante períodos de hasta 72 horas después de su ingestión $(1,2,16)$.

Durante las primeras horas tras la ingestión de estos alimentos este fenómeno se debe a la eructación, pero sucesivamente es consecuencia de la volatilización en el aire alveolar, a través de la circulación pulmonar, de metabolitos malolientes. Tales compuestos circulan en el torrente sanguíneo una vez que han sido absorbidos y metabolizados (1), y su excreción también puede realizarse a través de la saliva $(1,2)$.

La ingestión de té, café y especialmente de vino, licores y cerveza (bebidas que contienen ésteres solubles del alcohol y polifenoles) constituye una causa común de halitosis, reconocible de manera tan fácil como típica $(1,13,16)$.

Además, la ingestión de bebidas alcohólicas, así como la utilización frecuente de colutorios que contengan alcohol, puede provocar hiposialia y sequedad bucal, factores que, como queda dicho, favorecen la producción de compuestos volátiles malolientes (49).

\section{Humo de tabaco}

También el humo de tabaco, tanto de cigarrillo como de pipa o cigarro, aporta al aliento de los sujetos expuestos un olor característico y persistente (1). Curiosamente, muchas personas, en un pasado reciente, solían fumar para disimular problemas de halitosis, práctica que no hace más que generar un mal olor con el fin de enmascarar otro (8).

El olor a cigarrillo puede persistir durante más de un día después de haber fumado (8), y el mismo humo pasivo puede alterar el aliento de sujetos no fumadores $(1,8)$.

Algunos investigadores han valorado la posibilidad de que el humo de tabaco pueda incrementar la síntesis de compuestos volátiles de sulfuro en pacientes con enfermedad periodontal. En el estudio de Khaira y colaboradores (50), llevado a cabo sobre 23 pacientes con periodontopatía (12 fumadores y 11 no fumadores), los resultados han detectado, efectivamente, una correlación significativa entre producción de CVS y tabaquismo. Las causas de dicho efecto aún no están completamente aclaradas, y por el momento sólo se han formulado algunas hipótesis: el humo del tabaco podría alterar el equilibrio microbiano de la placa subgingival, determinando un aumento de las especies productoras de compuestos malolientes, y podría crear condiciones de anaerobiosis que, como se ha apuntado, favorecen el metabolismo putrefactivo y la consiguiente liberación de CVS. 
Cabe recordar también que, al igual que el consumo de bebidas alcohólicas, también el tabaco provoca hiposialia y reduce la lubricación de la cavidad oral (49).

\section{Fármacos}

Algunos fármacos pueden provocar situaciones transitorias de halitosis cono efecto secundario indeseado de la farmacocinética, debido a catabolitos terminales liberados en la circulación sistémica y eliminados a través de diferentes vías, entre ellas la pulmonar.

Los fármacos cuyo efecto en la generación de halitosis es conocido son el sulfóxido de dimetilo, que puede proporcionar al aliento un olor similar al del ajo; nitratos como el dinitrato de isosorbida; los compuestos que contienen yoduro y algunos medicamentos citostáticos, que pueden generar un olor dulzón (1, 2, 46) (tabla 6).

La utilización prolongada de antibióticos puede determinar foetor oris como consecuencia del cambio selectivo de la población microbiana de la cavidad oral (13).

La reducción del flujo salival provocada por algunas terapias farmacológicas (fármacos antineoplásicos, antidepresivos, antipsicóticos, narcóticos, descongestionantes, antihistamínicos, antihipertensivos y diuréticos) influye negativamente en la halitosis, reduciendo las capacidades de autolimpieza y favoreciendo, por consiguiente, la proliferación de biopelículas bucales $(1,14,46,51)$.

Especialmente los pacientes bajo tratamiento quimioterápico antineoplásico pueden presentar, debido a la acción citotóxica y mielosupresiva de los fármacos, candidiasis oral, sangrado gingival y úlceras bucales, factores todos ellos que desencadenan la halitosis (46).

\section{Causas fisiológicas}

Entre las causas fisiológicas, algunos autores han correlacionado la halitosis con el ciclo menstrual
TABLA 6.- MEDICAMENTOS IMPLICADOS EN LA PRODUCCIÓN DE MAL OLOR. ADAPTADA DE: FRACCARI F. BOGINI A.

COCCHETTO R. Alitosi E FOETOR EX ORE. STOMAT LOMB 1988:2(2);295-303.

\begin{tabular}{|l|l|}
\hline Medicamentos & Aplicación \\
\hline Nitroderivados & Angina \\
\hline Hidrato de cloral & Sedación \\
\hline $\begin{array}{l}\text { Compuestos con } \\
\text { yoduros }\end{array}$ & Expectorantes mucolíticos \\
\hline Antihistamínicos & Alergia, sedación \\
\hline Antineoplásicos & Terapia de tumores \\
\hline Diuréticos & $\begin{array}{l}\text { Antihipertensivos, } \\
\text { antiinflamatorios }\end{array}$ \\
\hline $\begin{array}{l}\text { Fenotiacinas } \\
\text { y sus derivados }\end{array}$ & $\begin{array}{l}\text { Esquizofrenia, psicosedantes, } \\
\text { antieméticos }\end{array}$ \\
\hline Tranquilizantes & Sedación \\
\hline Anfetaminas & Analépticos \\
\hline $\begin{array}{l}\text { Sulfóxido } \\
\text { de dimetilo }\end{array}$ & Dolor muscular, cistitis \\
\hline Noxiflex & Antiséptico \\
\hline
\end{tabular}

(aliento menstrual) (13): en un estudio de 1978 citado varias veces en la literatura $(2,3,8,9,46)$, Tonzetich comprobó el cambio de los niveles de CVS en el aire expirado durante el ciclo menstrual.

También resulta bastante frecuente la detección de halitosis en sujetos que emplean durante mucho tiempo la voz por exigencias profesionales, como es el caso de maestros, profesores y oradores.

\section{HALITOFOBIA}

La halitosis imaginaria o halitofobia es un trastorno de origen psicopatológico en el que el paciente acu- 
sa una presencia persistente de mal olor en su aliento no detectable objetivamente por el observador (1).

Los pacientes aquejados de halitofobia consideran inútiles e improductivos los tratamientos recomendados para la corrección de la halitosis, como es el caso de la adopción de medidas oportunas de higiene oral o alimentaria, y recurren con frecuencia a varios especialistas, sometiéndose a numerosas investigaciones clínicas (1). Atribuyen significados erróneos a comportamientos ajenos como el alejamiento relativo del interlocutor durante una conversación, el gesto de taparse la cara por parte del mismo, o a particulares actitudes de la mímica facial, como si tales acciones estuvieran causadas por la percepción del olor desagradable de su aliento $(1,52,53)$.

Los sujetos halitofóbicos pueden dividirse en dos subgrupos principales: individuos aquejados de "halitosis imaginaria" $(1,53)$ o "pseudohalitosis" (52), en los que efectivamente no se da una detección objetiva del mal olor del aliento, y sujetos aquejados de halitosis, a la que se añaden fuertes tendencias a la psicosomatización sistémica del trastorno.

En el primer caso, el paciente no acepta como plausible la explicación de que su convicción carece de fundamento, mientras que los pacientes pertenecientes a la segunda tipología no creen que su problema pueda reducirse o incluso eliminarse tras los tratamientos locales prescritos, y persisten en acusar la presencia continua de su síntoma $(1,53)$.

Un estudio de Rosenberg y colaboradores (8) ha delineado las principales características psíquicas de los pacientes halitofóbicos: se trata de sujetos con complejos de inferioridad acentuados, extremadamente emotivos y escasamente propensos a las relaciones sociales; algunos de ellos manifiestan características ansioso-depresivas y, en los casos más graves, actitudes obsesivo-compulsivas. Algunos individuos pueden presentar o desarrollar tendencias depresivas (2); su estilo de vida se ve fuertemente condicionado por su trastorno, lo que los lleva con frecuencia al aislamiento social y, en los casos más graves, a propósitos suicidas $(1,2,16,52)$.

Los pacientes aquejados de halitofobia intentan evitar toda situación de contacto social, se cepillan los dientes y comen caramelos continuamente y se mantienen siempre a una "distancia de seguridad" durante su conversación con las demás personas (8).

El tratamiento de estos pacientes resulta complejo, y precisa la valoración y la intervención de un psicólogo o de un especialista en psiquiatría (1).

A la hora de tratar a estos pacientes no conviene olvidar, sin embargo, que pueden manifestarse alucinaciones gustativas y olfativas en sujetos aquejados de esquizofrenia, epilepsia, tumores cerebrales $\mathrm{y}$, en ocasiones, en pacientes con lesiones cerebrales orgánicas $(2,46)$.

La incapacidad de percibir correctamente el propio olor no es únicamente una característica exclusiva de los pacientes halitofóbicos: muy al contrario, es frecuente el caso de individuos aquejados de halitosis que no están en condiciones de reconocer y evaluar su propio aliento. En un estudio posterior de Rosenberg y colaboradores (54) se visitó a 43 pacientes que referían estar aquejados de halitosis. Se les enseñaron procedimientos de autoevaluación con el fin de verificar cuál era el grado de percepción de su problema. Como cabía esperar, durante la primera consulta las diferencias entre los resultados de la autoevaluación y los de la evaluación organoléptica llevada a cabo por jueces expertos resultaban significativas, pero los resultados más interesantes se obtuvieron en la convocatoria de un año después. Pese a que los pacientes habían sido instruidos acerca de las causas de la halitosis, de cuáles terapias debían adoptar y de cómo evaluar el mal olor, y aunque su problema había mejorado en la gran mayoría de ellos, sólo el 35\% reconoció que se había registrado una mejoría en las propias condiciones.

El estudio puso, pues, en evidencia que la autoevaluación de los niveles de olor en individuos con halitosis está determinada por concepciones previas que no se vuelven objetivas con el avance de la experiencia y del tiempo.

En conclusión, se trata de personas que tienden a emitir juicios más subjetivos y son incapaces de evaluar eventuales mejorías tras terapias adecuadas, mientras que los individuos no aquejados de halitosis o que suponen que no lo están, una vez instrui- 
dos acerca de las causas de la patología y de sus eventuales terapias, desarrollan mayores capacidades objetivas de autoevaluación (54).

\section{CONCLUSIONES}

La revisión de la literatura ha puesto de relieve una correlación entre el foetor ex ore y algunos hábitos dietéticos y de ocio, así como con algunas patologías locales o sistémicas.

Siendo de todos conocido lo difícil que resulta propiciar el cambio de hábitos reiterativos alimentarios y de ocio incluso ante patologías bastante más graves, aún más ardua se revelará la tarea del odontólogo y del higienista dental a la hora de motivar al paciente aquejado de halitosis para que interrumpa el consumo de café, bebidas alcohólicas, ajo, cebollas o incluso carne, huevos y productos queseros, sin olvidar el tabaquismo activo y pasivo: factores todos ellos significativamente correlacionados con el foetor ex ore.

La atención del odontólogo y del higienista dental deberá centrarse en reducir la incidencia de los factores de su competencia que desencadenan la halitosis, ciñéndose especialmente a la eliminación de toda concausa posible que favorezca la retención de restos alimenticios y de la placa bacteriana y al mantenimiento de la salud de la cavidad oral.

Más concretamente, el higienista dental deberá indicarle al paciente las causas principales y, por consiguiente, las metodologías de eliminación de las mismas que se revelen indispensables con vistas a un tratamiento correcto de la halitosis. El higienista actuará motivando al paciente para que recurra al hilo dental y al cepillado de los dientes, sin olvidar el de la lengua, particularmente el de su tercio posterior. El frotamiento de la superficie lingual tiene como objetivo eliminar el cubrimiento, lo que reduce drásticamente la carga bacteriana con la consiguiente disminución en la producción de compuestos volátiles de sulfuro (23). La lengua podrá limpiarse con un cepillo suave o con un instrumento específico para ese fin; procederá recomendarle al paciente que actúe con delicadeza para evitar lesiones a las mucosas y para minimizar el reflejo del vómito. Adi- cionalmente a las maniobras mecánicas de eliminación de la placa dentogingival y del cubrimiento lingual, el higienista podrá recomendar como coadyuvantes en el tratamiento de la halitosis el uso de colutorios a base de gluconato de clorhexidina al $0,2 \%$ durante breves períodos y de colutorios que contengan listerina, cloruro de cetilpiridinio, dióxido de cloro, aceites esenciales, triclosán o zinc durante períodos prolongados $(1,2,4,7,11,13,15$, $16,20,29,41,49)$. Por el contrario, el uso de dentífricos, pastillas y chicles publicitados como productos contra el mal aliento sólo se ha demostrado eficaz durante unas horas después de su aplicación (2, 4, 17, 34, 51).

En cualquier caso, la halitosis sigue constituyendo una ocasión más de contacto entre el paciente y el equipo odontológico que el higienista debe necesariamente utilizar para reconducir al paciente a la motivación de la salud oral y con el fin de insertarlo en un programa de controles periódicos, en cuyo marco será posible poner en evidencia y tratar, en colaboración con el odontólogo, todo aspecto de la patología odontoestomatológica que pudiera surgir.

\section{BIBLIOGRAFÍA}

1. Abati S. Alitosi. Eziopatogenesi, diagnosi e trattamento. Milano: Ed. Masson; 2001.

2. Scully C, El-Maaytah M, Porter SR. Breath odor: etiopathogenesis, assessment and managernent. Eurj Oral Sci 1997;105:287-93.

3. Miyazaki H, Sakao S, Katoh Y, Takehara T. Correlation between votatile sulphur compound and certain oral health. measurements in the general population. J Periodontol 1995;66:67984.

4. Loesche WJ, Kazor C. Microbiology and treatment of halitosis. 2000 Periodontol 28:25679.

5. Eli I, Bath R, Koriat H, Rosemberg M. Selfperception of breath odor. J Am Dent Assoc 2001 May;132:621-6. 
6. Söder B, Johansson B, Söder PO. The relation between foetor ex ore, oral hygiene and periodontal disease. Swed Dent J 2000;24:73-82.

7. Loesche WJ. The effect of antimicrobiat mouthrinses on oral matodor and their status relative to US Food and Drug Administration regulations. Quintessence Int 1999 May;30:311-8.

8. Rosemberg M. Clinical assessment of bad breath: current concepts. J Am Dent Assoc 1996 Apr; 127:475-82.

9. Neiders $M$, Ramos B. Operation of bad breath clinics. Quintessence Int 1999;30:295-301.

10. Delanghe G, Ghyselen MD, Bollen C et al. An inventory of patients' response to treatment at a multidisciplinary breath odor clinic. Quintessence Int 1999;30:307-10.

11. Rösing CK, Jonski G, Rolla. Comparative analysis of some mouthrinses on the production of volatile sulfur-containig compounds. Acta Odontol Scand 2002;60:10-2.

12. Sterer $N$, Greenstein $R$, Rosemberg $M$. Galactosidase activity in saliva is associated with oral malodor. J Dent Res 2002;81(3):182-5.

13. Touyz L. Oral malodor: a scientific perspective. Can Dent Assoc 1993;S9:607-10.

14. Lenton P, Majerus G, Bakdash B. Counseting and treating bad breath patients: a step by step approach. J Contemp Dent Pract 2001;2(2):46-61.

15. Frascella J, Gilbert RD, Fernandez P, Hendler J. Efficacy of a chlorine dioxide containing mouthrinse in oral malodor. Compend Contin Educ Dent 2000;21(3):241-6.

16. Fischman SL. Over the counter mouthrinses. J Calif Dent Assoc 1998;26(3):204-6.

17. Greenstein RB, Goldberg S, Cohen S, Sterer N, Rosemberg M. Reduction of oral malodor by oxidizing lozenges. J Periodontol 1997;68: 1176-81.
18. Sánz M, Roldan S, Herrera D. Fundamentals of breath malodour. J Contemp Dent Pract 2001; $4(2): 1-17$.

19. Quirynen M, Mongardini C, van Steenberghe D. The effect of a 1-stage full-mouth disinfection on oral malodor and microbial colonization of the tongue in periodontitis patients. A pilot study. $\mathrm{J}$ Periodontol 1998;69:374-82.

20. ADA council on scientific affair. Oral malodor. J Am Dent Assoc 2003;134(2):209-14.

21. De Boever EH, Loesche WJ. Assesing the contribution of anaerobic microflora of the tongue to oral malodor. J Am Dent Assoc 1995, 126:1384-93.

22. Ratcliff PA, Johnson PW. The relationship between oral malodor, gingivitis and periodontitis. J Periodontol 1999;70:458-89.

23. Seeman R, Kison A, Bizhang M, Zimmer S. Effectivness of mechanical tongue cleaning on oral levels of volatile sulfur compounds, J Am Dent Assoc 2001;132:1263-7.

24. Morita M, Wang HL. Association between oral malodor and adult periodontitis: a review. J Clin Periodontol 2001;28:813-9.

25. Kleinberg I, Codipilly M. Modeling the oral malodor system and method of analysis. Quintessence Int 1999;30:357-69.

26. Shimura M, Qwatanabe S, lwakura $M$ et al. Correlation between measurements using a new halitosis monitor and organoleptic assessment. J Periodontol 1997;68:1182-5.

27. Waler SM. On the transformation of sulfurcontainig amino acids and peptides to volatile sulfur compounds (VSC. in the human mouth. Eur J Oral Sci 1997;105:534-7.

28. Tonzetich J. Production and origin of oral malodor: a review of mechanism and method of analysis. J Periodontol 1977;48(1):13-20. 
29. Ritcher JL. Diagnosis and treatment of halitosis. Compend Contin Educ Dent 17(4):370-86.

30. Pera P, Genovesi AM, Romano L, Nardi G. Alitosi: un problema orate diffuso. Prevenzione e assistenza dentale 2000;4:20-4.

31. Nachnani S. Oral malodor: a detailed review. CHDA Jounal 1999.

32. Moss SJ. Halitosis and oral malodor. Report submitted to the FDI Commission 1998;maggio:1-6.

33. Bosy A, Kulkarni GV, Rosemberg M, McCulloch CA, Relationship of oral malodor to periodontitis: evidence of independence in discrete subpopulations. J Periodontol;65:37-46.

34. Reigewirtz Y, Girault O, Reingewirtz N, Senger B, Tenebaum H. Mechanical effects and volatile sulfur compound reducing effects of chewing gums: comparison between test and base gums and a control goup. Quintessence Int 1999; 30:319-23.

35. Kazor CE, Mitchell PM, Lee AM, Stokes LN, Loesche WJ, Dewhirst E, Paster BJ. Diversity of bacterial populations on the tongue dorsa of patients with halitosis and healthy patients. J Clin Microb 2003;41(2):558-63.

36. Yaegaki K, Sanada K. Biochemical and clinical factors influencing oral malodor in pariodontal patients. J Periodontol 1992;63:783-9.

37. Rosenberg M Kulkarni GV, Bosy A. McCulloch CA. Reproducibility and sensitivity of oral malodor measurements with a portable sulfide monitor. J Dent Res 1991;70:1436-40.

38. Kleinberg I. Westbay M. Salivary and metabolic factors involved in oral malodor formation. $J$ Periodontol 1992;63(9):768-75.

39. Figueiredo LC, Rosetti EP, Marcantonio E, Rosemary A, Salvador SL. The relationship of oral malodor in patients with or wlthout periodontal disease. J Periodontol 2002;73:133842.
40. Tonzetich J. Oral malodour: an indicator of health status and oral cleanliness. Int Dent 1978; 28(3):309-19.

41. Kozlovsky A, Goldberg S, Natour I, Gat A, Rosemberg M. Efficacy of a 2-phase oil water mouthrinse in controlling oral malodor, gingivitis and plaque. J Periodontol 1996;67:577-82.

42. Golberg S, Cardash H, Browning H, Sahly H, Rosenberg $M$. Isolation of enterobacteriaceae from the mouth and potential association with malodor. J Dent Res 1997;76(11):1770-5.

43. Myatt GJ, Hunt SA, Barlow AP, Winston L, Ashley PB, Bordas A, El Maaytah M. A clinical study to asses the breath protection efficacy of denture adhesive. J Contemp Dent Pract 2002;4(3):1-9.

44. Fornabaio AM, Marziliano P, Scagnetto E, Bocconi S. Lálitosi come problema nella societá moderna: considerazioni e previsioni per il futuro. 1 Congresso Nazionale M Docenti di Odontoiatria. Roma, 24-27 marzo 1994.

45. Preti G, Clark L, Beverly J, Feldman 5, Lowry LD, Weber E, Young IM. Non oral etiologies of oral malodor and altered chemosensation. J Periodontol 1992;63:790-6.

46. Fraccari F, Bogini A, Cocchetto R. Alitosi e foetor ex ore. Stomat Lomb 1988;2(2):295-303.

47. Yaegaki K, Sanada K. Volatile sulfur compounds in mouth air from clinically healthy subjects and patients with periodontital disease. J Periodontal Res 1992;27:233-8.

48. Norfleet RG. Helicobacter halitosis. J Clin Gastroenterol 1993,16:274.

49. The history of professional formulas utilizing stabilized chlorine dioxide for efficacious treatment of bad breath. 4th Internetional Conference on Oral malodor. UCLA School of Dentistry, August 1999.

50. Khaira N, Palmer RM, Wilson RF, Scott DA, Wade WG. Production of volatile sulphur compounds 
in diseased periodontal pockets is significantly increased in smokers. Oral Disease 2000;6: 371-5.

51. Sharma NC, Galustians HJ, Qaquish J, Galustians A, Rustogi KN, Petrone ME, Chaknis P, Garcia L, Volpe AR, Proskin HM. The clinical effectivness of a dentifrice containing triclosan and a copolymer for controlling breath odor measured organoleptically twelve hours after toothbrushing. J Clin Dent 1999;10:131-4.

52. Yaegaki K, Coil MJ. Examination, classification, and treatment of halitosis; clínical perspectives. J Can Dent Assoc 2000;66:257-61.
53. Yaegaki K, Coil M. Clinical dilemmas posed by patients with psychosomatic halitosis. Quintessence Int 1999;30:328-33.

54. Rosemberg M, Kozlovsky A, Wind Y, Mindel E. Self-assessment of oral malodor 1 year following initial consultation. Quintessence Int 1999; 30:324-7.

55. Lang NP, Attström R, Löe H. Proceedings of the European Workshop on mechanical plaque control. Berne, Switzerland, 9-12 maggio.

56. Rosenberg M, McCulloch CA. Measurement of oral malodor: current methods and future prospects. J Periodontol 1992;63:776-82. 